\title{
A motivational framework of social comparison Supplementary Material
}

October 11, 2020

\section{Supplementary Figure 1: Domain Frequencies}

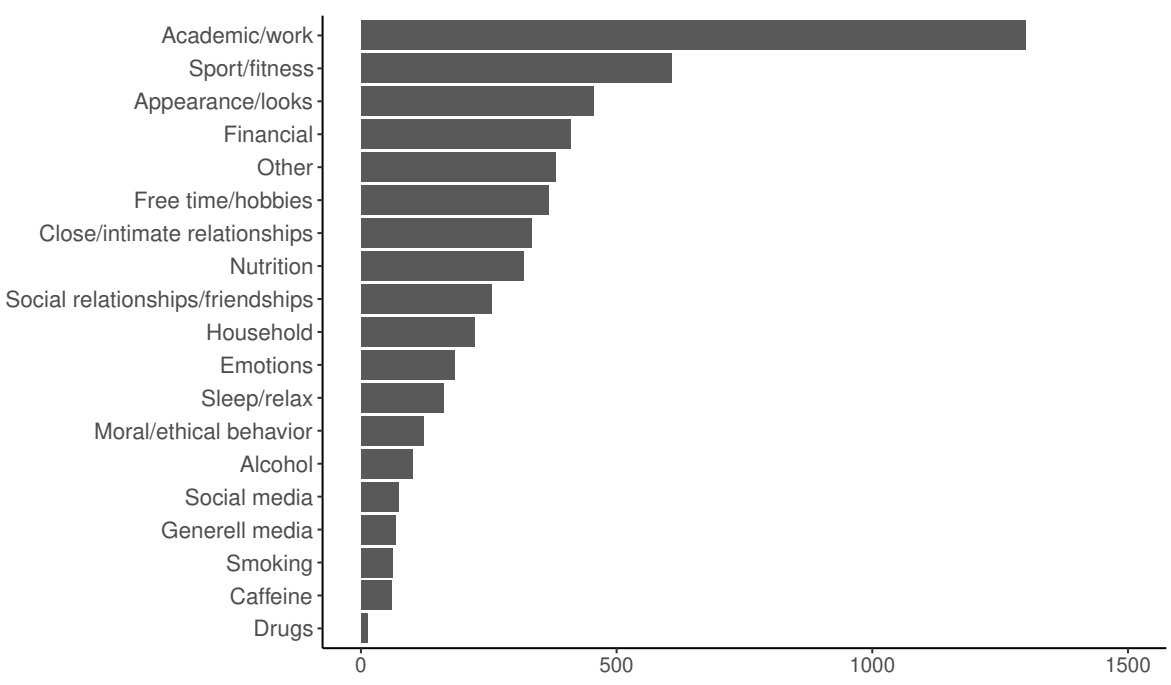

Figure 1: Social comparison frequencies in all domains 
2 Supplementary Figure 2: Effort Investment

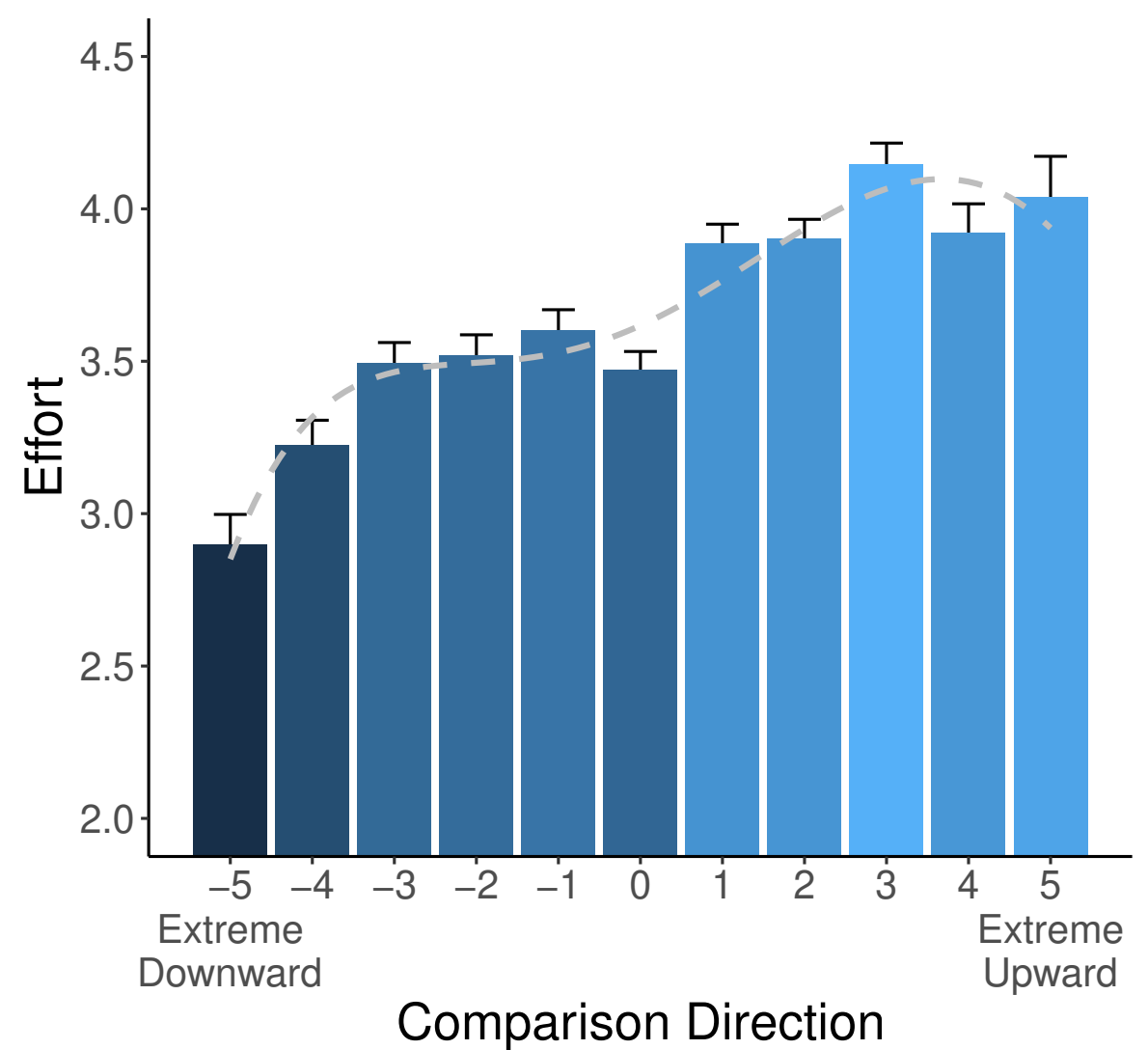

Figure 2: Effects of comparison direction on effort investment. The dotted grey line shows the estimated curve from the multilevel polynomial regression analysis.

\section{Moderation Analyses}

\subsection{Domain Control}

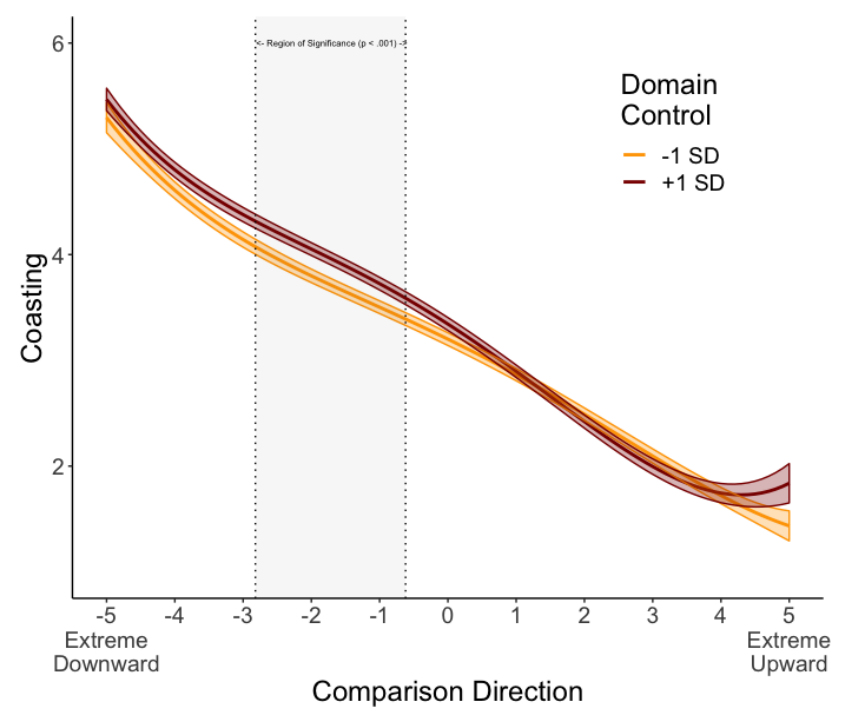

Figure 3: Coasting predicted by multilevel polynomial regression and their interaction effects with high vs. low domain control. 
Table 1: Multilevel Polynomial Regression Table of 1) the Effects of Comparison Direction, Domain Control and their Interactions and 2) Deviance Model Tests comparing a Nested Base Model to the Interaction Model

\begin{tabular}{|c|c|c|c|c|c|}
\hline & \multirow[b]{2}{*}{ Variable } & \multicolumn{2}{|c|}{ Model 1} & \multicolumn{2}{|c|}{ Model 2} \\
\hline & & $B$ & $p$ & $B$ & $p$ \\
\hline \multirow[t]{11}{*}{ Pushing } & (constant) & 4.35 & $<.001$ & 4.35 & $<.001$ \\
\hline & control & 0.22 & $<.001$ & 0.24 & $<.001$ \\
\hline & direction & 0.14 & $<.001$ & 0.14 & $<.001$ \\
\hline & direction $^{2}$ & 0.03 & .002 & 0.03 & .001 \\
\hline & direction $^{3}$ & 0.00 & .724 & 0.00 & .508 \\
\hline & direction $^{4}$ & 0.00 & $<.001$ & 0.00 & $<.001$ \\
\hline & direction $*$ control & & & 0.04 & .006 \\
\hline & direction $^{2} *$ control & & & 0.00 & .941 \\
\hline & direction $^{3} *$ control & & & 0.00 & .447 \\
\hline & direction ${ }^{4} *$ control & & & 0.00 & .633 \\
\hline & \multicolumn{5}{|c|}{ Deviance Model Test with: $\chi^{2}(4)=23.23, p<.001$} \\
\hline \multirow[t]{11}{*}{ Disengagement } & (constant) & 1.86 & $<.001$ & 1.86 & $<.001$ \\
\hline & control & -0.08 & $<.001$ & -0.05 & .023 \\
\hline & direction & 0.19 & $<.001$ & 0.19 & $<.001$ \\
\hline & direction $^{2}$ & 0.03 & $<.001$ & 0.04 & $<.001$ \\
\hline & direction $^{3}$ & 0.00 & .060 & 0.00 & .220 \\
\hline & direction $^{4}$ & 0.00 & .615 & 0.00 & .867 \\
\hline & direction $*$ control & & & 0.01 & .341 \\
\hline & direction $^{2} *$ control & & & 0.00 & .570 \\
\hline & direction $^{3} *$ control & & & 0.00 & .063 \\
\hline & direction $^{4} *$ control & & & 0.00 & .708 \\
\hline & \multicolumn{5}{|c|}{ Deviance Model Test with: $\chi^{2}(4)=10.77, p=.029$} \\
\hline \multirow[t]{11}{*}{ Coasting } & (constant) & 3.27 & $<.001$ & 3.27 & $<.001$ \\
\hline & control & 0.04 & .001 & 0.05 & .018 \\
\hline & direction & -0.36 & $<.001$ & -0.37 & $<.001$ \\
\hline & direction $^{2}$ & -0.02 & .006 & -0.03 & .002 \\
\hline & direction $^{3}$ & 0.00 & .512 & 0.00 & .747 \\
\hline & direction $^{4}$ & 0.00 & $<.001$ & 0.00 & $<.001$ \\
\hline & direction $*$ control & & & & \\
\hline & direction $^{2} *$ control & & & -0.01 & .287 \\
\hline & direction $^{3} *$ control & & & 0.00 & .006 \\
\hline & direction $^{4 *}$ control & & & 0.00 & .172 \\
\hline & \multicolumn{5}{|c|}{ Deviance Model Test with: $\chi^{2}(4)=14.01, p=.007$} \\
\hline
\end{tabular}




\subsection{Domain Importance}

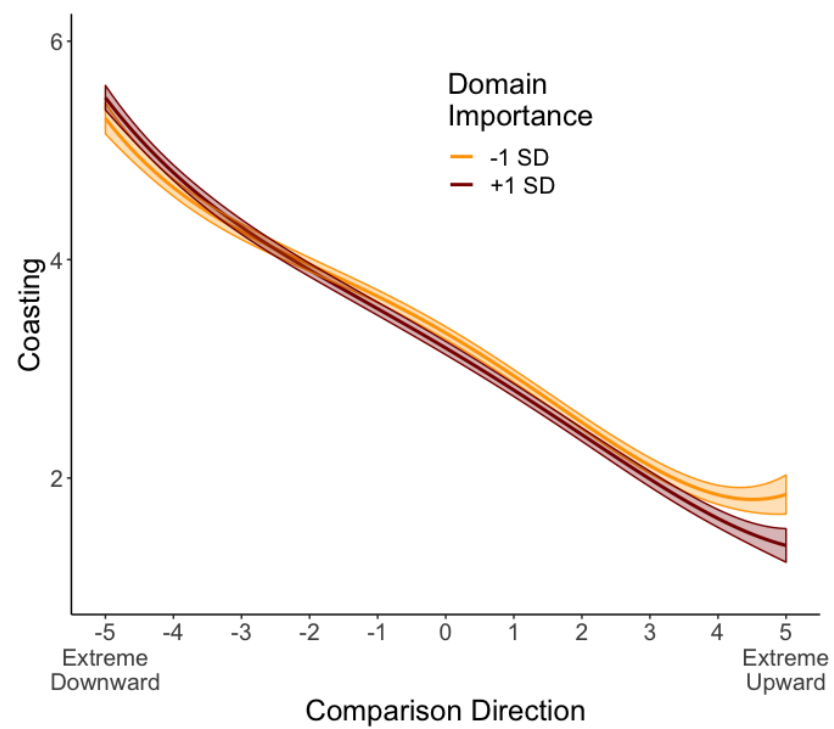

Figure 4: Coasting predicted by multilevel polynomial regression and their interaction effects with high vs. low domain importance. 
Table 2: Multilevel Polynomial Regression Table of 1) the Effects of Comparison Direction, Domain Importance and their Interactions and 2) Deviance Model Tests comparing a Nested Base Model to the Interaction Model

\begin{tabular}{|c|c|c|c|c|c|}
\hline & \multirow[b]{2}{*}{ Variable } & \multicolumn{2}{|c|}{ Model 1} & \multicolumn{2}{|c|}{ Model 2} \\
\hline & & $B$ & $p$ & $B$ & $p$ \\
\hline \multirow[t]{11}{*}{ Pushing } & (constant) & 4.45 & $<.001$ & 4.46 & $<.001$ \\
\hline & importance & 0.42 & $<.001$ & 0.49 & $<.001$ \\
\hline & direction & 0.13 & $<.001$ & 0.13 & $<.001$ \\
\hline & direction $^{2}$ & 0.01 & .363 & 0.01 & .501 \\
\hline & direction $^{3}$ & 0.00 & .445 & 0.00 & .224 \\
\hline & direction $^{4}$ & 0.00 & $<.001$ & 0.00 & $<.001$ \\
\hline & direction $*$ importance & & & 0.03 & .056 \\
\hline & direction $^{2} *$ importance & & & -0.01 & .077 \\
\hline & direction $^{3} *$ importance & & & 0.00 & .948 \\
\hline & direction $^{4} *$ importance & & & 0.00 & .579 \\
\hline & \multicolumn{5}{|c|}{ Deviance Model Test with: $\chi^{2}(4)=34.25, p<.001$} \\
\hline \multirow[t]{11}{*}{ Disengagement } & (constant) & 1.87 & $<.001$ & 1.87 & $<.001$ \\
\hline & importance & 0.04 & .001 & 0.00 & .847 \\
\hline & direction & 0.19 & $<.001$ & 0.20 &.$<.001$ \\
\hline & direction $^{2}$ & 0.03 & $<.001$ & 0.04 & $<.001$ \\
\hline & direction $^{3}$ & 0.00 & .032 & 0.00 & .125 \\
\hline & direction $^{4}$ & 0.00 & .579 & 0.00 & .841 \\
\hline & direction $*$ importance & & & 0.00 & .865 \\
\hline & direction $^{2} *$ importance & & & 0.01 & .252 \\
\hline & direction $^{3} *$ importance & & & 0.00 & .025 \\
\hline & direction ${ }^{4} *$ importance & & & 0.00 & .886 \\
\hline & \multicolumn{5}{|c|}{ Deviance Model Test with: $\chi^{2}(4)=31.27, p<.001$} \\
\hline \multirow[t]{11}{*}{ Coasting } & (constant) & 3.26 & $<.001$ & 3.26 & $<.001$ \\
\hline & importance & -0.03 & .020 & -0.06 & .014 \\
\hline & direction & -0.37 & $<.001$ & -0.37 & $<.001$ \\
\hline & direction $^{2}$ & -0.02 & .010 & -0.02 & .010 \\
\hline & direction $^{3}$ & 0.00 & .424 & 0.00 & .706 \\
\hline & direction $^{4}$ & 0.00 & $<.001$ & 0.00 & $<.001$ \\
\hline & direction $*$ importance & & & 0.00 & .839 \\
\hline & direction $^{2} *$ importance & & & 0.01 & .268 \\
\hline & direction $^{3} *$ importance & & & 0.00 & .201 \\
\hline & direction $^{4} *$ importance & & & 0.00 & .308 \\
\hline & \multicolumn{5}{|c|}{ Deviance Model Test with: $\chi^{2}(4)=10.62, p=.031$} \\
\hline
\end{tabular}




\subsection{Active Comparison Seeking}

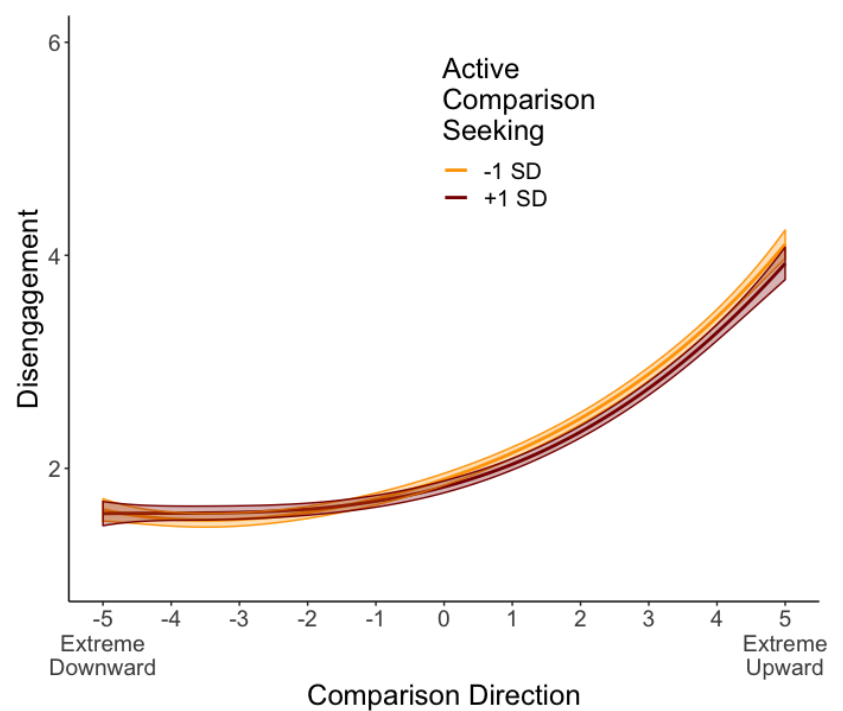

Figure 5: Multilevel Polynomial Regression Table of 1) the Effects of Comparison Direction, Active Comparison Seeking and their Interactions and 2) Deviance Model Tests comparing a Nested Base Model to the Interaction Model 
Table 3: Multilevel Polynomial Regression Table: Effects of Comparison Direction, Active Comparison, and their Interaction on Pushing, Disengagement and Coasting

\begin{tabular}{|c|c|c|c|c|c|}
\hline & \multirow[b]{2}{*}{ Variable } & \multicolumn{2}{|c|}{ Model 1} & \multicolumn{2}{|c|}{ Model 2} \\
\hline & & $B$ & $p$ & $B$ & $p$ \\
\hline \multirow[t]{11}{*}{ Pushing } & (constant) & 4.34 & $<.001$ & 4.34 & $<.001$ \\
\hline & active & 0.11 & $<.001$ & 0.11 & $<.001$ \\
\hline & direction & 0.13 & $<.001$ & 0.12 & $<.001$ \\
\hline & direction $^{2}$ & 0.03 & .005 & 0.03 & .004 \\
\hline & direction $^{3}$ & 0.00 & .335 & 0.00 & .355 \\
\hline & direction $^{4}$ & 0.00 & $<.001$ & 0.00 & $<.001$ \\
\hline & direction $*$ active & & & 0.02 & .074 \\
\hline & $\operatorname{direction}^{2} *$ active & & & 0.00 & .971 \\
\hline & $\operatorname{direction}^{3} *$ active & & & 0.00 & .253 \\
\hline & direction ${ }^{4} *$ active & & & 0.00 & .987 \\
\hline & \multicolumn{5}{|c|}{ Deviance Model Test with: $\chi^{2}(4)=4.14, p=.388$} \\
\hline \multirow[t]{11}{*}{ Disengagement } & (constant) & 1.86 & $<.001$ & 1.86 & $<.001$ \\
\hline & active & -0.02 & .034 & -0.03 & .129 \\
\hline & direction & 0.19 & $<.001$ & 0.19 & $<.001$ \\
\hline & direction $^{2}$ & 0.04 & $<.001$ & 0.04 & $<.001$ \\
\hline & direction $^{3}$ & 0.00 & .031 & 0.00 & .046 \\
\hline & direction $^{4}$ & 0.00 & .727 & 0.00 & .774 \\
\hline & direction $*$ active & & & -0.02 & .043 \\
\hline & $\operatorname{direction}^{2} *$ active & & & 0.00 & .540 \\
\hline & $\operatorname{direction}^{3} *$ active & & & 0.00 & .381 \\
\hline & $\operatorname{direction}^{4} *$ active & & & 0.00 & .484 \\
\hline & \multicolumn{5}{|c|}{ Deviance Model Test with: $\chi^{2}(4)=8.25, p=.083$} \\
\hline \multirow[t]{11}{*}{ Coasting } & (constant) & 3.27 & $<.001$ & 3.27 & $<.001$ \\
\hline & active & 0.01 & .539 & 0.02 & .230 \\
\hline & direction & -0.36 & $<.001$ & -0.37 & $<.001$ \\
\hline & direction $^{2}$ & -0.02 & .005 & -0.02 & .005 \\
\hline & direction $^{3}$ & 0.00 & .391 & 0.00 & .445 \\
\hline & direction $^{4}$ & 0.00 & $<.001$ & 0.00 & $<.001$ \\
\hline & direction $*$ active & & & 0.01 & .253 \\
\hline & $\operatorname{direction}^{2} *$ active & & & 0.00 & .336 \\
\hline & $\operatorname{direction}^{3} *$ active & & & 0.00 & 639 \\
\hline & direction $^{4} *$ active & & & 0.00 & .348 \\
\hline & \multicolumn{5}{|c|}{ Deviance Model Test with: $\chi^{2}(4)=3.55, p=.470$} \\
\hline
\end{tabular}




\section{Controlling for Domain Competence}

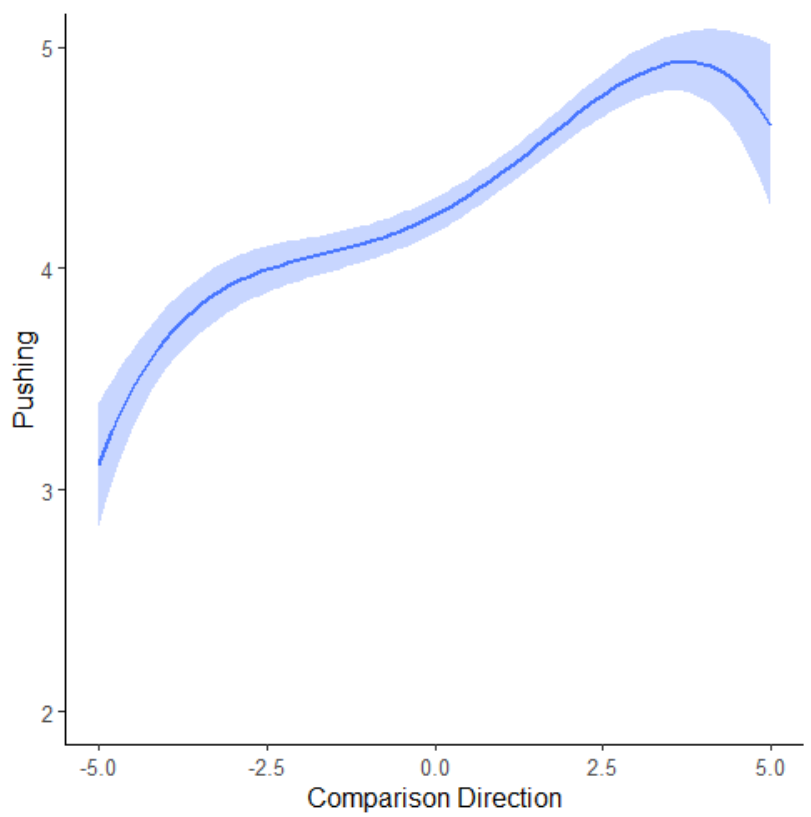

(a) Pushing

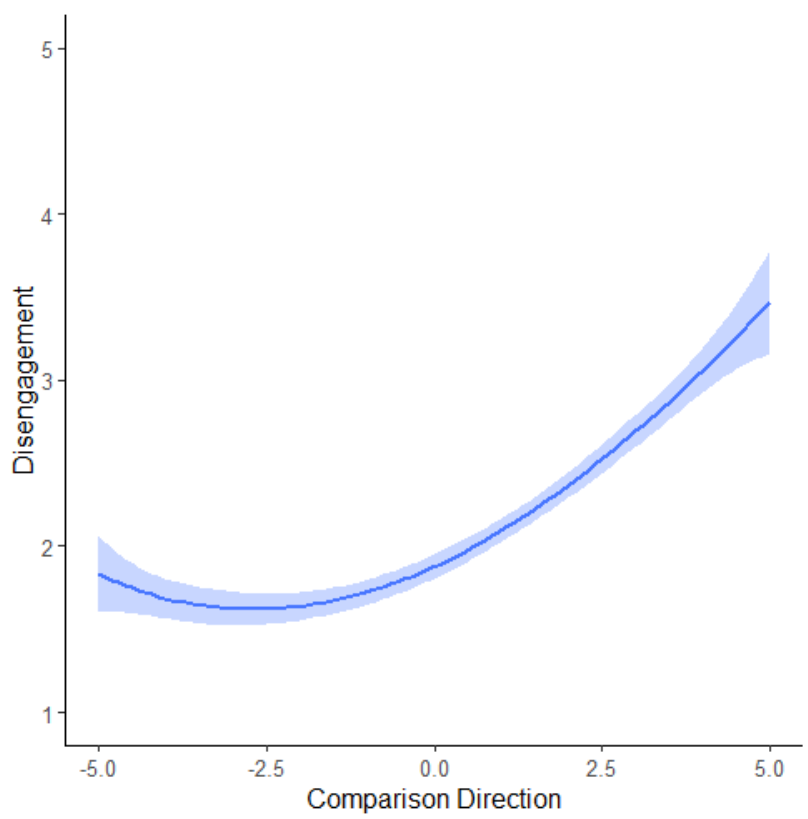

(b) Disengaging

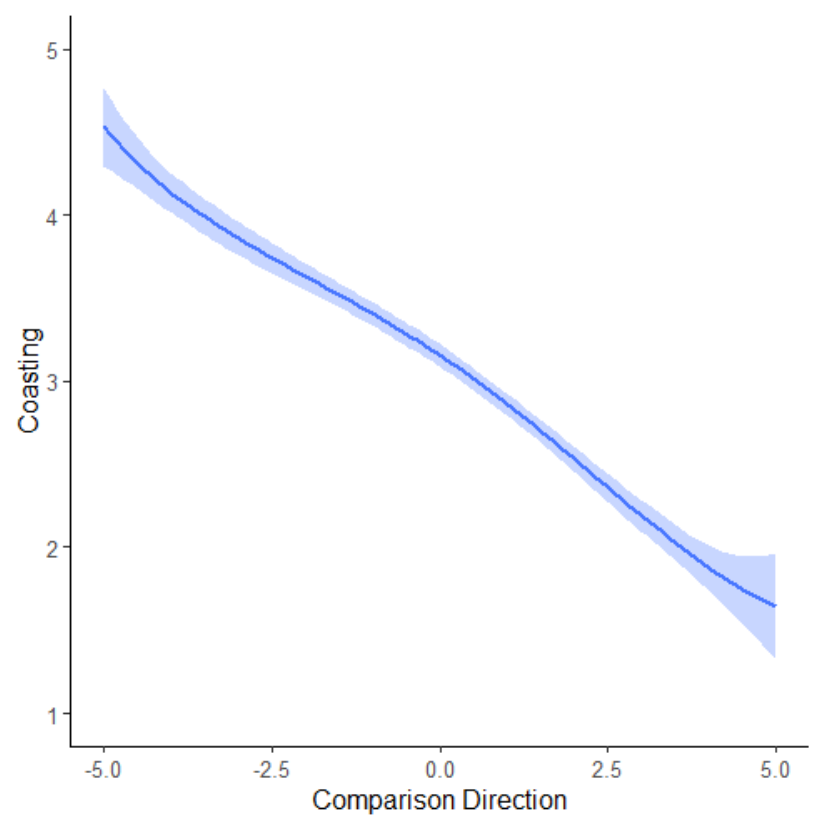

(c) Coasting

Figure 6: Estimated curves at midpoint (4) of domain competence scale (nobs = 1,382); i.e., domain competence "kept constant" at midpoint). 